\title{
Cage Effects in Recombination of Allyl Radicals in Irradiated Polyethylene. II. Cumulative Reaction Rates and Decay Kinetic Processes
}

\author{
LIAN-PIN HWANG, Department of Chemistry, National Taiwan \\ University, Taipei, Taiwan, Republic of China
}

\begin{abstract}
Synopsis
Hwang and Cheng have studied the recombination reaction of allyl radicals in irradiated polyethylene by including the effects of (i) diffusion of macroradicals by jumps of finite size in the crystalline phase and (ii) a caging reaction with a finite rate in the disordered region. In this work their results are used to analyze cumulative reaction rate data on the decay of allyl radicals in extendedchain and Marlex film polyethylene. The kinetic parameters obtained show the effects of reaction temperature, irradiation dose, and morphological differences.
\end{abstract}

\section{INTRODUCTION}

The decay of radicals in irradiated polymer has been treated in terms of Waite's equation, which is based on the Brownian motion mechanism of the Smoluchowski model. ${ }^{1}$ Rather large values of the encounter distance (10-80 $\AA$ ) have been obtained. The long-range encounter distance and subsequent reaction processes remain unexplained. ${ }^{2}$ It has been shown experimentally that the overall kinetics in solid polymer may be resolved approximately into an initial stage with a "fast" reaction rate followed by a normal stage with a reduced rate. One explanation for the occurrence of a two-stage reaction involving different rates in the amorphous and crystalline phases of the irradiated polymer ${ }^{3-6}$ is that of Dole et al., who proposed kinetics involving two simultaneous second-order reactions occurring with and without diffusion control in different zones for allyl radical decay in polyethylene and obtained two reaction rate constants, $k_{f}$ and $k_{s}$, to represent the fast and the slow processes, respectively. However, the $k_{f}$ values show no definite trend either with temperature or amorphous content, in contrast to the behavior of $k_{s}$. They also tried to apply Waite's equation to interpret the decay kinetics of allyl radicals in extended-chain polyethylene. . $^{3,4}$ However, it should be noted that Waite's equation only applies to the system at long times. ${ }^{7}$ Thus the significance of their results is doubtful, and the linear dependence of the reciprocal concentration of allyl radicals on the square root of time may be accidental. Furthermore, molecular diffusion in solid polymer should not be expected to obey Waite's equation, which is adequate only for Brownian molecules, ${ }^{7}$ since molecular motion in the polymer matrix is severely hindered, especially in the presence of entangled and crosslinked polymer molecules. Hence, unlike the motion of a Brownian molecule, which continuously takes small steps, radical diffusion is expected to be characterized by the time interval $\tau_{j}$ between the two successive steps due to hindrance. 
Recently, in Part I of this series ${ }^{8}$ Hwang and Cheng presented a cage model to take into account the recombination processes of radicals in solid polymers. To describe the diffusion hindrance, they applied the jump-diffusion model of Torrey ${ }^{9}$ to the evaluation of time-dependent reactivity with a radiation boundary condition to simulate cage reactions with finite rates. The motivation is the fact that the decay of radicals occurs mostly in disordered (amorphous) regions. ${ }^{10}$ Thus these regions can be considered as reaction cages. In the crystalline phase the segmental diffusion is more or less inhibited and radical sites, which participate in the slow reaction process, migrate from the crystalline phase to cages. It is suggested that cage recombination requires two radicals to remain inside a cage for a certain period of time until random motion of the macromolecular chain leads finally to diffusion or combination of the macroradicals. As a consequence, the diffusion coefficient obtained from kinetic measurements characterizes only diffusion in the crystalline phase of the sample. The mean time $\tau_{j}$ between jumps becomes longer at lower temperature and/or for higher irradiation doses. Since increasing the irradiation dose produces more crosslinks, the mobility of chain segments containing allyl radicals decreases. Moreover, the temperature dependence of cage size and the intrinsic rate of the cage reaction may reflect the motion of macromolecules in the disordered (amorphous) phase.

It is our purpose here to apply the theory developed previously to interpret the decay kinetics of allyl radicals in irradiated extended-chain and Marlex polyethylene films.4,5 A further objective of this study is to examine the effects of changes in irradiation dose and temperature on the measured kinetic parameters. This may reveal the importance of such effects in polymer diffusion and in the cage reaction as well. In addition, morphological aspects are considered in relation to the present results.

\section{REACTION SYSTEM AND FORMALISM}

To describe the model described above, we calculate the observed reaction rate $k_{t}$, which is determined by the rate equation

$$
-\frac{d C}{d t}=k_{t} C^{2}
$$

where $C$ is the concentration of allyl radicals at time $t$. The integration of eq. (1) relates directly to the experimental observation of changes in reciprocal concentration in the reaction, i.e.,

$$
\frac{1}{C}-\frac{1}{C_{0}}=\int_{0}^{t} k_{t^{\prime}} d t^{\prime}
$$

where $C_{0}$ is the initial concentration of allyl radicals. As shown by Hwang, ${ }^{7,11}$ the observed reaction rate is given by

$$
k_{t}=k_{\text {in }} V P(d, t)
$$

with

$$
p(d, t)=\frac{1}{8 \pi^{2} V} \dot{\int} d \Omega \int_{v} d^{3} \mathbf{r}_{0} P\left(\mathbf{r}_{0} \mid \mathbf{d}, t\right)
$$


where $V$ is the volume of the system and the integrals are evaluated over the initial uniform distribution and the entire range of solid angle of the caging sphere with radius $d$. It suffices for the present purpose to consider the reaction medium isotropic and the initial distribution of allyl radicals uniform, since it is reasonable to believe that allyl radicals exist in both the crystal core and on the surfaces. They are produced by reaction of alkyl radicals with double bonds located in both the crystal core and crystal surfaces. ${ }^{12}$ The intrinsic reaction rate $k_{\text {in }}$ may be related to the quantities defined by

$$
k_{\text {in }} \equiv 4 \pi d^{2} h D
$$

where $D$ is the sum of the diffusion coefficients of the individual reacting species, i.e., $D \equiv D_{A}+D_{B}$. The general vector form $P\left(\mathbf{r}_{0} \mid \mathbf{r}, t\right)$ is the conditional probability related to the diffusion-influenced reaction of molecules $A$ and B; i.e., given that they are separated by $\mathbf{r}_{0}$ at time $t=0$, the probability that they are separated by $\mathbf{r}$ at time $t$ with radiation boundary condition at the cage radius $d$ :

$$
\left.\frac{\partial P\left(\mathbf{r}_{0} \mid \mathbf{r}, t\right)}{\partial r}\right|_{r=d}=h P\left(\mathbf{r}_{0} \mid \mathbf{d}, t\right)
$$

where we define $h$ to represent the cage reactivity.

It was assumed in Torrey's theory that a particle performs jumps such that the individual jump vectors in space are statistically independent and have identical distributions with a probability density $P_{1}(\mathbf{r})$. The relative positions of the particles, after $n$ jumps, can be obtained by noting that the characteristic function of the final distribution is equal to $A^{n}[\rho]$, where

$$
\begin{aligned}
A[\rho] & =\int P_{1}(\mathbf{r}) \exp (i \boldsymbol{\rho} \cdot \mathbf{r}) d^{3} \mathbf{r} \\
& =\frac{1}{1+D \tau_{j} \rho^{2}}
\end{aligned}
$$

where $\tau_{j}$ is related to the mean-square jump distance $\left\langle r^{2}\right\rangle$ by $D \tau_{j}=1 / 6\left\langle r^{2}\right\rangle$. The diffusion model described above corresponds to a wave-vector diffusion equation with a $\rho$-space-dependent diffusion coefficient, ${ }^{7,13}$ i.e.,

$$
\frac{\partial P[\rho, t]}{\partial t}=-\rho^{2} D[\rho] P[\rho, t]
$$

where

$$
D[\rho] \equiv D A[\rho]
$$

and $P[\rho, t]$ is the Fourier transform of $P\left(\mathbf{r}_{0} \mid \mathbf{r}, t\right)$. The appropriate solution with the radiation boundary condition is given elsewhere ${ }^{7,9}$ and is not given in detail here. Substituting the solution $P\left(\mathbf{r}_{0} \mid \mathbf{r}, t\right)$ into eq. (4) and performing the integrations, we obtain the cumulative reaction rate, eq. (2), via eq. (3), e.g.,

$$
\begin{aligned}
\int_{0}^{t} k_{t^{\prime}} d t^{\prime}= & \frac{k_{\text {in }}}{1+h d}\left\{\frac{h d}{\gamma}[1-\exp (-\gamma t)]+t\right. \\
& +\frac{h d t}{\left(\tau_{j} / \tau\right)^{1 / 2}(1+h d)} \exp \left(\frac{-t}{2 \tau_{j}}\right)\left[I_{0}\left(\frac{t}{2 \tau_{j}}\right)+I_{1}\left(\frac{t}{2 \tau_{j}}\right)\right] \\
& \left.-\frac{h d t}{\left(\tau_{j} / \tau\right)^{1 / 2}(1+h d)} \Phi_{2}\left(1 / 2,1,2 ;-\frac{t}{\tau_{j}},-\gamma t\right)\right\}
\end{aligned}
$$


TABLE I

Experimental Conditions and Parameters Utilized in Curves E1-E3 and M1-M3

\begin{tabular}{|c|c|c|c|c|c|c|c|c|}
\hline Sample & $\begin{array}{c}\text { Temper- } \\
\text { ature } \\
\left({ }^{\circ} \mathrm{C}\right)\end{array}$ & $\begin{array}{c}\text { Dose } \\
\text { (Mrad) }\end{array}$ & $\begin{array}{c}d \\
(\AA)\end{array}$ & $\begin{array}{c}D \\
\left(\mathrm{~cm}^{2} / \mathrm{s}\right. \\
\left.\times 10^{18}\right) \\
\end{array}$ & $\begin{array}{c}\tau_{j} \\
(\min )\end{array}$ & $\begin{array}{c}\left\langle r^{2}\right\rangle^{1 / 2} \\
(\AA)\end{array}$ & $h d$ & $\begin{array}{c}k_{\text {in }} \\
\left(\mathrm{cm}^{3} / \mathrm{s}\right. \\
\left.\times 10^{22}\right) \\
\end{array}$ \\
\hline E1 & 120 & 7.6 & 25.0 & 10.42 & 10.0 & 19.4 & 5 & 1.64 \\
\hline $\mathrm{E} 2$ & 120 & 18.6 & 18.2 & 2.98 & 11.2 & 11.0 & 13 & 0.89 \\
\hline E3 & 120 & 39.8 & 18.5 & 1.64 & 7.0 & 6.4 & 20 & 0.76 \\
\hline M1 & 110 & 39.8 & 30.0 & 13.73 & 4.4 & 14.1 & 13 & 6.72 \\
\hline M2 & 100 & 39.8 & 23.0 & 6.49 & 6.9 & 12.7 & 18 & 3.38 \\
\hline M3 & 80 & 39.8 & 17.5 & 2.96 & 10.4 & 10.5 & 14 & 0.91 \\
\hline
\end{tabular}

a Note that samples E1-E3 are extended-chain polyethylene and samples M1-M3 are Marlex film polyethylene.

where $I_{n}(x)$ is the $n$ th-order modified Bessel function, $\Phi_{2}$ is the hypergeometric function of two variables, ${ }^{14}$ and $\gamma$ is given by

$$
\gamma \equiv\left[\tau_{j}-\tau /(1+h d)^{2}\right]^{-1}
$$

where $\tau \equiv d^{2} / D$. By means of eqs. (2) and (9), radical diffusion processes of various morphological properties may be better visualized.

\section{COMPARISON WITH EXPERIMENT}

To get the results described below, the observed cumulative reaction rate is examined by evaluating the integral representation ${ }^{7,8}$ of the hypergeometric

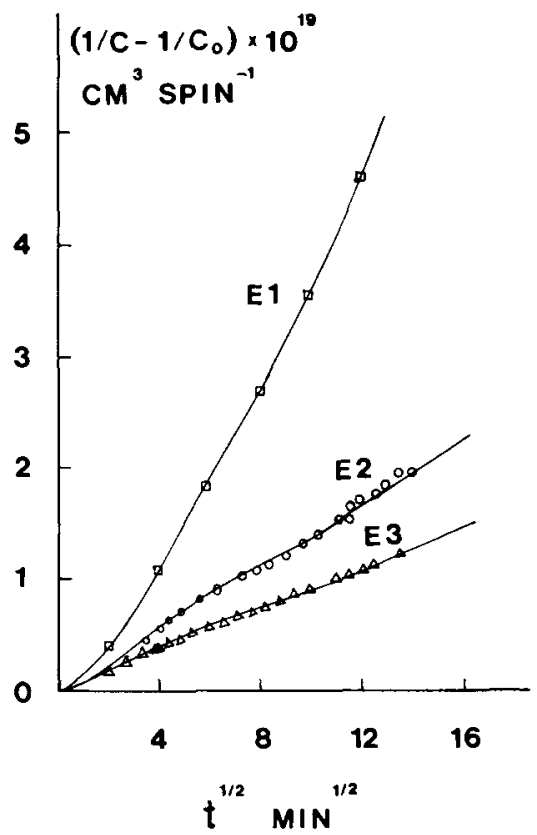

Fig. 1. Comparison of experimental results of Dole et al. with theoretical calculations for decay of allyl radicals in extended-chain polyethylene. Curve fitting parameters are listed in Table I. 


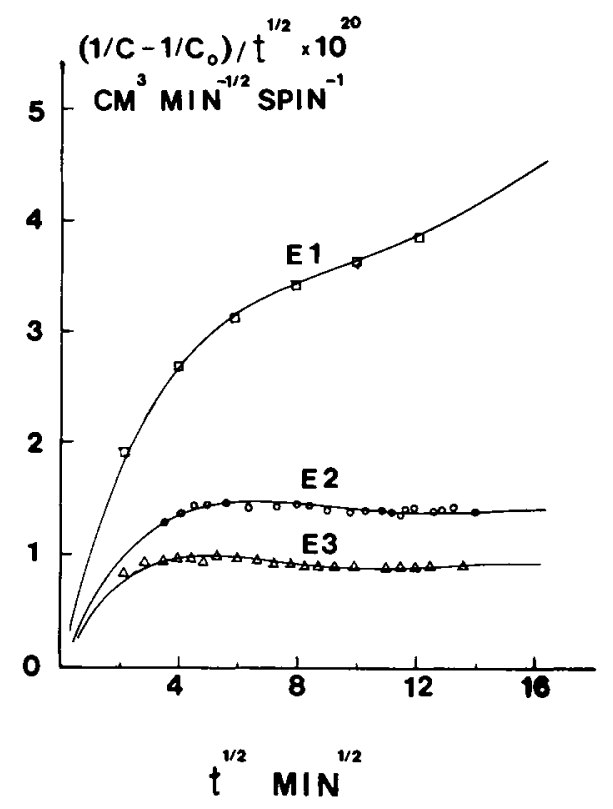

Fig. 2. The same data as in Fig. 1 but plotted as $\left(1 / C-1 / C_{0}\right) / t^{1 / 2}$ vs. $t^{1 / 2}$. Details are given in the text.

equation. The remaining integral is evaluated numerically. The details of curve fitting have already been discussed elsewhere. 8 The parameters used in fitting experimental data are listed in Table I. The matching of the change in allyl radical concentration change is reasonable if the least-squares error between data and theoretical curve and the wide spread of data points in the $t / \tau$ domain are taken into account in determining the optimal theoretical curve. We find that no other distinct set of parameters can fit the same experimental curve equally well.

In Figures 1 and 2 representative data obtained by Dole et al. on extendedchain polyethylene are plotted as $1 / C-1 / C_{0}$ and $\left(1 / C-1 / C_{0}\right) / t^{1 / 2}$ vs. $t^{1 / 2}$. The latter method of plotting was suggested by Wen et al. ${ }^{3}$ to reduce the error in the initial time measurement. It allows one to determine the magnitude of $\tau_{j}$ more accurately. The data used here are at $120^{\circ} \mathrm{C}$ with 7.6-, 18.6-, and 39.8-Mrad doses. The solid curves are calculated theoretically according to eq. (2). All the fitting parameters are listed in Table I. It is found that a larger diffusion coefficient gives rise to a drastic decrease of radical concentration in sample E1 as compared with samples receiving larger irradiation doses. In the latter more crosslinks are produced, which would hinder the movement of chain segments to a greater extent and so inhibit diffusion and subsequent recombination of the macroradicals. Figures 3 and 4 depict similar plots for Marlex film with a 39.8-Mrad dose at 80,100 , and $110^{\circ} \mathrm{C}$. It is seen that the fit between experiment and theory is satisfactory except for the data at $100^{\circ} \mathrm{C}$ beyond $120 \mathrm{~min}$. It is interesting to examine the temperature dependence of the kinetic parameters. At higher temperatures, since the segment mobility is greater and diffusion is less hindered, $\tau_{j}$ is expected to be smaller whereas the diffusion coefficient and the cage radius show opposite trends. The temperature-dependent diffusion 


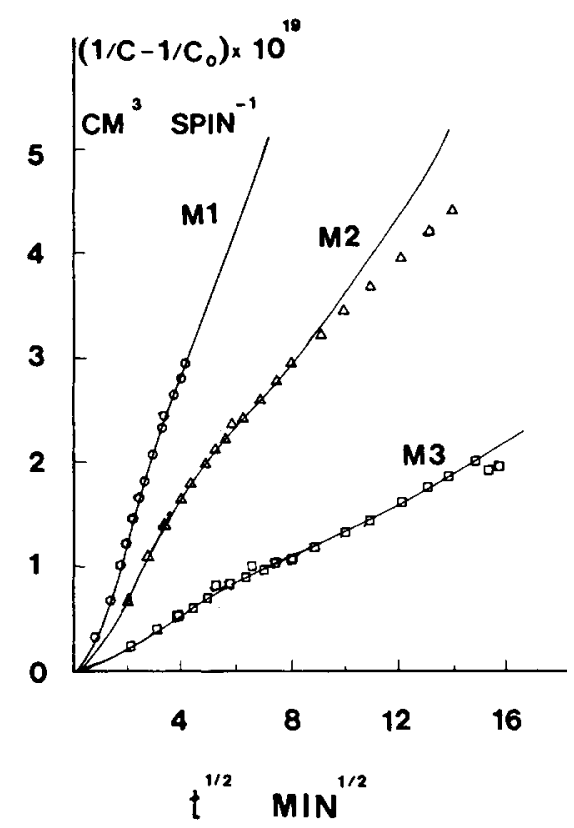

Fig. 3. Comparison of experimental results of Dole et al. with theoretical calculations for decay of allyl radicals in Marlex film polyethylene. Curve fitting parameters are listed in Table I.

coefficients for Marlex film give an activation energy $E_{D}=13.1 \mathrm{kcal} / \mathrm{mol}$, which is smaller than the value obtained before. ${ }^{8}$ The activation energy $E_{d}$ for the thermal variation of cage radius $\left(E_{d} \approx 4.6 \mathrm{kcal} / \mathrm{mol}\right.$ ) coincides with the previous result. $^{8}$ It corresponds to an activation energy of $14 \mathrm{kcal} / \mathrm{mol}$ for the thermal

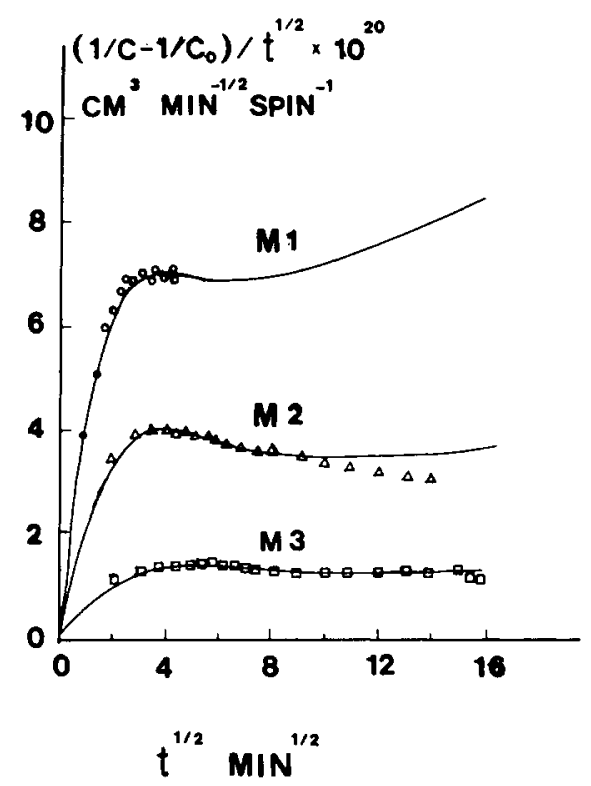

Fig. 4. The same data as in Fig. 3 but plotted as $\left(1 / C-1 / C_{0}\right) / t^{1 / 2}$ vs. $t^{1 / 2}$. Details are given in the text. 
expansion of cage volume. This value is consistent with the activation energy of free-volume formation measured at high temperature for most polymers. ${ }^{15}$ However, it should be noted that the activation energy of free-volume formation becomes very large at temperatures near the glass temperature, and values in excess of $200 \mathrm{kcal} / \mathrm{mol}$ for polymers have been reported. ${ }^{15}$ Thus the activation energy obtained in this study is valid at high temperatures only.

The obtained mean-square jump distances show definite dependences on temperature and irradiation dose; i.e., the mean-square jump distances increase with temperature and decrease with increasing irradiation dose. This is expected since crosslinks within each single crystal and some crosslinks in defects within the crystal of polyethylene have been found. Therefore one may infer that not only the surfaces of crystals but also the defects within crystals offer cages for radical reactions between adjacent chains. Furthermore, the diffusion coefficient is much smaller in extended-chain polyethylene than in Marlex film. This may be attributed to slower diffusion in lamellae with the extremely large thickness achieved in the formation of extended-chain crystals.

In Table I are also shown the effects of temperature and irradiation doses on the intrinsic reaction rate: higher temperature leads to a significant increase of $k_{\text {in }}$, while higher irradiation doses decrease it. The variation in $k_{\text {in }}$ may be explained by the effects of temperature-dependent chain mobility and cage radius. It has an activation energy of $17.7 \mathrm{kcal} / \mathrm{mol}$, which is approximately the sum of $E_{d}$ and $E_{D}$. The same conclusion can be drawn as well from the previous study, i.e., values of $h d$ are found to be only weakly temperature dependent, and the intrinsic reaction rate is mainly controlled by the temperature dependence of cage size and diffusion coefficient.

\section{CONCLUSION}

We have shown in this work how the kinetic theory of the jump diffusion model allows us to interpret the decay of allyl radicals in extended-chain and Marlex film polyethylene. Kinetic parameters were determined from the best fit between theoretical curves and experimental points. The application of a cage model here and in the previous preliminary paper provides a satisfactory description of the experimental data on various types of polyethylene. The most general feature of the present model is to treat the macroradical diffusion in the crystalline phase and the subsequent cage reaction in the disordered regions. Some aspects of the present results favor Flory's "switchboard" model ${ }^{16}$ of chain conformation at the fold surface, since it allows, in the disordered region, a greater degree of segmental motion, which plays a dominant role in the recombination reaction of allyl radicals in the cage. Also, a general tendency for cage size to increase with increasing temperature or decreasing irradiation dose supports the validity of the cage reaction model.

Finally, we note that the refinements in our analysis of diffusion kinetics are in conformity with the experimental results. While the treatment is idealized in some repects, the close correspondence to the experimental observations will be useful in analyzing data on radical decay in solid polymers. The development of a method for estimating the kinetic parameters in recombination reactions is in progress and will be published elsewhere.

Grateful acknowledgment is made of support of the work reported here by the National Science Council of the Republic of China. 


\section{References}

1. T. R. Waite, Phys. Rev., 107, 463 (1957).

2. P. Yu Butyagin, Vysokomol. Soedin. Ser. A, A16, 63 (1974).

3. W. Y. Wen, D. R. Johnson, and M. Dole, J. Phys. Chem., 78, 1978 (1974).

4. M. Dole and J. Salik, J. Am. Chem. Soc., 99, 6454 (1977).

5. V. M. Patel, G. N. Patel, N. Gvozdic, C. S. Hsu, and M. Dole, J. Polym. Sci. Polym. Phys. Ed., 16, 467 (1978).

6. R. Basheer and M. Dole, Int. J. Chem. Kinet., 13, 1143 (1981).

7. L. P. Hwang, J. Chem. Phys., 76, 4037 (1982).

8. L. P. Hwang and J. T. Cheng, J. Polym. Sci. Polym. Phys. Ed., 19, 983 (1981), Part I of this series.

9. H. C. Torrey, Phys. Rev., 92, 962 (1953).

10. M. Kryszewski, B. Nadolski, and M. Zujewicz, J. Polym. Sci. Polym. Symp., 57, 129 (1976).

11. L. P. Hwang, J. Chem. Phys., 73, 183 (1980).

12. T. Fujimura, N. Hayakawa, and I. Kuriyama, J. Polym. Sci. Polym. Phys. Ed., 16, 945 (1978).

13. G. T. Evans and M. Fixman, J. Phys. Chem., 80, 1544 (1976).

14. (a) G. E. Roberts and H. Kaufman, Table of Laplace Transforms, Saunders, Philadelphia, 1966; (b) A. Erdelyi, Higher Transcendental Functions, McGraw-Hill, New York, 1953, Vol. 1, p. 225 .

15. F. Bueche, Physical Properties of Polymers, Interscience, New York, 1970, p. 90.

16. P. J. Flory and D. Y. Yoon, Nature, 272, 226 (1978).

Received December 6, 1982

Accepted May 2, 1983 